\title{
Bilateral patellar tendon rupture
}

\author{
Kiran Divani, Padmanabhan Subramanian, Konstantinos Tsitskaris, David Crone and \\ Mandeep Lamba \\ Trauma and Orthopaedics Department, Whipps Cross University Hospital, Leytonstone, London EII INR, UK \\ Corresponding author: Kiran Divani. Email: divanik@doctors.org.uk
}

Although uncommon, bilateral patellar tendon rupture is preposterously debilitating. Ultrasonography is valuable in diagnosing patellar tendinosis and preventing subsequent rupture.

\section{Case report}

We present an exceedingly rare case of spontaneous bilateral patellar tendon rupture in a fit and well 38year-old Afro-Caribbean gentleman regularly participating in recreational long-distance running. Prior to sustaining this injury, he complained of ongoing anterior knee pain, for which he was awaiting an outpatient X-ray arranged by his general practitioner after weeks of anti-inflammatory medications proved ineffective. The patient was jogging and sustained a fall onto his right knee. The patient was unable to return to his feet, requiring assistance to hospital. He presented to the Accident and Emergency department with pain, swelling and lack of active extension at both knees. Clinically, there was swelling at the patellar tendons, with a highriding patellar bilaterally, confirmed on plain radiography.

He was subsequently admitted under the care of the orthopaedic team where he was surgically managed with simultaneous primary repair. Midline incisions were made over both knees, and repair was achieved using Krackow pull-out sutures with fibre wire, ethibond-modified kesseler suture, fibre glass box suture and retinacular repair with vicryl 1-0. The Insol Selvesti ratio was checked as 1:1 on both sides under image intensifier. Finally, cylinder back slabs were applied bilaterally to immobilize both knee joints.

Postoperatively, the patient was transferred to the ward where he received a week of inpatient physiotherapy. Post-discharge, he was reviewed in the fracture clinic fortnightly where both wounds demonstrated healthy healing with no signs of infection or inflammation. At the seventh postoperative week, his casts were exchanged for hinged braces to initiate movement from $0-40^{\circ}$ and he was referred to the physiotherapist for quadriceps strengthening exercises with whom he made good progress.

\section{Discussion}

A Medline and Pubmed literature search reveals that there have been sporadic cases of bilateral patella tendon rupture, but this is the first ever case reported in a young fit and well gentleman without any past medical history or regular medications.

The knee extensor apparatus is commonly simplified. It is composed of the quadriceps muscles that converge into a central tendon in a three-layered arrangement with the rectus femoris superficial, vastus medialis and lateralis in the intermediate layer and the vastus intermedius most deeply. This attaches to and invests the patellar before continuing as the patellar tendon, which inserts into the tibial tuberosity allowing the leg to extend at the knee joint on contraction of these quadriceps muscles. ${ }^{1}$ This description, although accepted, does not take into account its complexity and variability with a study demonstrating that only three of 20 dissected specimens have exhibited this typically described pattern, while the remainder had bi and even more complex tri and tetralaminar fibre arrangements. ${ }^{2}$ Pathology can occur at any length of this kinetic chain creating a significant deficiency in functionality.

'Tendinitis' and 'tendinosis' are descriptive terms for tendon pathology, limited to suggest inflammation and tendon degeneration with a failed healing response, respectively. The umbrella term 'tendinopathy', coined by Maffuli et al. ${ }^{3}$ in 1998, is better suited for tendon pathology, used as a generic descriptor of conditions in and around tendons arising from overuse, encompassing pain, swelling and impaired performance. It has been successfully used for over a decade in accurately describing tendon pathology and minimizing the misuse of other descriptive terms. ${ }^{4}$

Patellar tendon rupture is an uncommon phenomenon but when occurring tends to have some 
Table I. Histopathological and radiological features suggestive of patellar tendinosis.

\begin{tabular}{|c|c|c|}
\hline \multirow[t]{2}{*}{ Radiology } & Ultrasound & MRI \\
\hline & $\begin{array}{l}\text { - Hypoechoic area in proximal patellar tendon } \\
\text { - Hypoechoic area combined with various amounts of } \\
\text { swelling in the surrounding tendon } \\
\text { - Hyperechoic regions considered to be calcification }\end{array}$ & $\begin{array}{l}\text { - Area of abnormal signal intensity within the } \\
\text { tendon at the junction with the patellar } \\
\text { - Lesions of increased signal intensity relative } \\
\text { to the tendon }\end{array}$ \\
\hline Histopathology & $\begin{array}{l}\text { - } \text { Pseudocyst change } \\
\text { - } \text { - Mucrinoid necrosis } \\
\text { - Randomized collagen with neovascularization and ten } \\
\text { - Microtears of the tendinous tissues } \\
\text { - Chronic inflammatory cell infiltration } \\
\text { - Focal degeneration near bone-tendon insertion } \\
\text { - Angiofibroblastic tendinosis }\end{array}$ & cyte infiltration \\
\hline
\end{tabular}

recognizable attributing factors. Some of these factors have been reported to include repeated corticosteroid injections into the patellar tendon, ${ }^{5}$ morbid obesity, ${ }^{6}$ jumping sports and sports involving prolonged and repetitive stress of the knee extensor apparatus. $^{7}$ Disease processes such as systemic lupus erythematosus, chronic renal failure, hyperparathyroidism and diabetes mellitus have also been implicated mainly via weakening of collagen fibres. ${ }^{8}$

Amidst the literature, within a sporting context, case reports on complete spontaneous rupture of almost every tendon possessed by the human body can be found. Achilles tendon rupture is the most closely associated with sporting activities. Patellar and quadricep tendon ruptures occur most often in the older population but in relation to sports is correlated to high-power events, such as high jump, basketball and weight lifting, mostly between the ages of 15-30 years. ${ }^{9}$

'Jumper's knee' or patellar tendinosis is a clinical entity predisposing to patellar tendon rupture. Various histopathological and radiological findings suggestive of Jumper's knee have been summarized by Khan et al. $(\text { Table } 1)^{10}$.

Knowledge of these diagnostic features is essential in the early recognition of patellar tendinosis. For this reason, practically feasible and cost-effective procedures such as ultrasound may have a role as a screening tool in aiding the prevention of patellar tendon rupture among the high-risk athletic community.

This case highlights some important points. First, although spontaneous bilateral patellar tendon rupture is uncommon, its occurrence can be preposterously debilitating. In this case, early recognition in the acute sector of the hospital enabled the patient to be referred to the appropriate specialty where contemporary surgical and rehabilitative techniques were provided, ensuring the best opportunity to restore his pre-morbid level of functional activity. However, in fit and well individuals with reports of anterior knee pain on weight-bearing activities, we feel ultrasonography can be valuable in diagnosing patellar tendinosis and preventing subsequent rupture.

\section{Declarations}

Competing interests: None declared

Funding: None declared

Ethical Approval: Written informed consent to publication has been obtained from the patient.

Guarantor: KD

Contributorship: All authors contributed equally.

Acknowledgements: None

Provenance: Submitted; peer reviewed by Ayaz Lakdawala

\section{References}

1. Dwek RJ and Chung CB. The patellar extensor apparatus of the knee. Paediatr Radiol 2008; 38: 925-935.

2. Waligora AC, Johanson NA and Hirsch BE. Clinical anatomy of the quadriceps femoris and extensor apparatus of the knee. Clin Orthop Relat Res 2009; 467: 3297-3306.

3. Maffuli N, Khan KM and Puddu G. Overuse tendon conditions: time to change a confusing terminology. J Arthrosc Related Surg 1998; 14: 840-843.

4. Divani K, Chan O, Padhiar N, et al. Site of maximum neovascularisation correlates with the site of pain in recalcitrant mid-tendon Achilles tendinopathy. Man Ther 2010; 15: 463-468.

5. Clark SC, Jones MW, Choudhury RR and Smith E. Bilateral patellar tendon rupture secondary to repeated local steroid injections. J Accid Emerg Med 1995; 12: 300-301. 
6. Panasiuk M and Groblewski M. Spontaneous patellar tendon rupture as a result of morbid obesity. Chir Narzadow Ruchu Ortop Pol 2011; 76: 353-354.

7. Hagglund M, Zwerver J and Ekstrand J. Epidemiology of patellar tendinopathy in elite male soccer players. Am J Sports Med 2011; 39: 1906-1911.

8. Kricun R, Kricun ME, Arangio GA, Salzman GS and Berman AT. Patellar tendon rupture with underlying systemic disease. AJR 1980; 135: 803-807.
9. Kannus P and Natri A. Etiology and pathophysiology of tendon ruptures in sports. Scand J Med Sci Sports 1997; 7: 107-112.

10. Khan KM, Bonar F, Desmond PM, et al. Patellar tendinosis (Jumper's knee): findings at histopathological examination, US, and MR imaging. Musculoskel Radiol 1996; 200: 821-827. 